\title{
Lithium Abundances in Solar-Type Stars ${ }^{1}$
}

\author{
L. da Silva \\ CNPq/Observatório Nacional, Brazil \\ G. F. Porto de Mello \\ Observatório do Valongo, Universidade Federal do Rio de Janeiro, Brazil
}

\begin{abstract}
We report $\mathrm{Li}$ abundances from the $\lambda 6707$ line for 19 nearby dwarf and subgiant solar-type stars. The unevolved stars in this sample present high $(>2.00) \mathrm{Li}$ abundances. We found a few cases of subgiant stars which present high Li content. The Sun seems to be part of a population of nearly unevolved stars which have depleted their $\mathrm{Li}$ to a high degree: all other metal-normal, near ZAMS stars in our sample show higher than solar Li content. There seems to be no correlation of the degree of $\mathrm{Li}$ depletion with mass, atmospheric parameters or state of evolution: as an example we found a star (HR1532) almost identical to the Sun in its state of evolution and atmospheric parameters, but with over ten times the solar $\mathrm{Li}$ abundance. We propose that different histories of angular momentum distribution at star birth, and/or post-birth angular momentum evolution, may account for these differences.
\end{abstract}

\section{Analysis and Results}

Observations have been performed at the $\mathrm{OPD}^{1}$, using the coudé spectrograph of the $1.60 \mathrm{~m}$ telescope was used to obtain $\mathrm{S} / \mathrm{N}>200,0.20 \AA$ resolution spectra of 19 solar-neighborhood, solar-type stars. The observed spectral regions cover approximately $100 \AA$ each, centered at $\lambda 6050, \lambda 6150$ and $\lambda 6707$. The Sun was observed as a star by way of lunar spectra, with the same setup. Atmospheric parameters $\mathrm{T}_{\text {eff }}, \log \mathrm{g},[\mathrm{Fe} / \mathrm{H}]$ and microturbulence velocities were obtained from the detailed analysis, differential with respect to the Sun, of the excitation \& ionization equilibria of $\mathrm{Fe}$ (over $25 \mathrm{Fe}$ I lines in average, $2 \mathrm{Fe}$ II lines). We have also derived log $\mathrm{g}$ from absolute magnitudes based on HIPPARCOS parallaxes: excellent agreement was obtained between the two approaches. Luminosities were obtained by applying the bolometric corrections of Habets \& Heintze (1981). Mean errors of the parameters thus obtained are: $70 \mathrm{~K}$ for the excitation $\mathrm{T}_{\text {eff }}$, 0.30 dex and 0.06 dex for, respectively, the ionization and evolutionary log $\mathrm{g}$; $0.07 \mathrm{dex}$ for $[\mathrm{Fe} / \mathrm{H}]$ and $0.15 \mathrm{~km} / \mathrm{s}$ for the microturbulent velocities. The lithium abundance was derived from the $\lambda 6707$ doublet by spectral synthesis of the ob-

\footnotetext{
${ }^{1}$ Based on observations collected at the Observatório do Pico dos Dias (OPD), Brazil, operated by the CNPq/Laboratório Nacional de Astrofísica .
} 
served line profiles, using a program kindly made available by Monique Spite (Observatoire de Paris- Meudon). The error in the determination is estimated as 0.10 dex.

Table 1. Atmospheric parameters, Fe, Li abundances and luminosities for the program stars.

$\begin{array}{rrrrrrrr}\mathrm{HR} & \mathrm{T}_{\text {eff }} & \log g_{\text {ion }} & \log g_{\text {evol }} & \xi_{k m / s} & {[\mathrm{Fe} / \mathrm{H}]} & \log \mathrm{L} / \mathrm{L}_{\odot} & \log \mathrm{N}(\mathrm{Li}) \\ 173 & 5270 & 3.75 & 3.84 & 1.35 & -0.70 & 0.48 & 0.00 \\ 914 & 5020 & 3.66 & 3.60 & 0.93 & -0.57 & 0.67 & 0.60 \\ 3138 & 5830 & 4.40 & 4.35 & 0.79 & -0.27 & 0.08 & 0.30 \\ 8501 & 5750 & 4.27 & 4.32 & 1.30 & -0.25 & 0.05 & 1.70 \\ 6998 & 5500 & 4.43 & 4.47 & 0.34 & -0.16 & -0.15 & -0.50 \\ 1747 & 5960 & 4.21 & 4.25 & 1.67 & -0.10 & 0.30 & 2.30 \\ 3862 & 6130 & 4.33 & 4.35 & 1.50 & -0.08 & 0.24 & 2.53 \\ 77 & 5970 & 4.48 & 4.46 & 1.08 & -0.07 & 0.07 & 2.30 \\ \mathrm{SUN} & 5780 & 4.44 & 4.44 & 1.30 & +0.00 & 0.00 & 1.15 \\ 3259 & 5380 & 4.38 & 4.45 & 1.07 & +0.00 & -0.19 & 0.30 \\ 2251 & 5950 & 4.36 & 4.32 & 1.47 & +0.01 & 0.19 & 2.05 \\ 695 & 5830 & 3.87 & 4.01 & 1.37 & +0.03 & 0.51 & 2.33 \\ 8635 & 5940 & 4.19 & 4.28 & 1.37 & +0.04 & 0.25 & 2.60 \\ 8323 & 5900 & 4.48 & 4.42 & 1.08 & +0.07 & 0.06 & 2.30 \\ 772 & 5710 & 4.00 & 4.05 & 1.53 & +0.09 & 0.43 & 1.70 \\ 1532 & 5740 & 4.36 & 4.47 & 1.06 & +0.09 & -0.03 & 2.25 \\ 810 & 6130 & 4.42 & 4.43 & 1.74 & +0.11 & 0.18 & 2.45 \\ 8700 & 5890 & 3.92 & 4.02 & 1.79 & +0.19 & 0.53 & 2.55 \\ 1536 & 5990 & 4.30 & 4.12 & 1.61 & +0.27 & 0.43 & 2.78 \\ 1856 & 6020 & 3.73 & 3.69 & 2.19 & +0.27 & 0.99 & 1.20\end{array}$

\section{Evolutionary State and Lithium Abundances}

We have examined the distribution of $\mathrm{Li}$ abundances with state of evolution by plotting the stars in the theoretical HR diagrams of Schaerer et al. (1993 and references therein), roughly corresponding to metallicities of $[\mathrm{Fe} / \mathrm{H}]=-0.37$, +0.03 and +0.33 . We show only the diagram corresponding to solar metallicity stars (figure 1). The metal-rich and metal-normal unevolved stars have preserved most of their $\mathrm{Li}$, in contrast to the Sun, which is the only unevolved Li-poor star. The Sun may thus be considered part of a population of stars which have strongly depleted their Li (Pasquini et al. 1994). It has been suggested by King et al. (1997) that different histories of Li depletion for stars with very similar masses may be linked to the formation of a planetary system: as an example they cite the binary system $16 \mathrm{Cyg} \mathrm{AB}$, both components being very solar-like (the $\mathrm{B}$ component being the planet harboring one), but differing in their Li content by a factor of $\sim 5$.We note that, in our sample, HR1532 is almost identical to the Sun in mass and atmospheric parameters but is more than ten times Li-richer than the Sun.

We have found a few cases of Li-rich subgiant stars. This may be understood by the "ressurgence" scenario (Dravins et al. 1993), in which the subgiant 
Figure 1. Theoretical HR diagrams for the near solar metallicity stars. labeled with HR numbers and Li abundances.

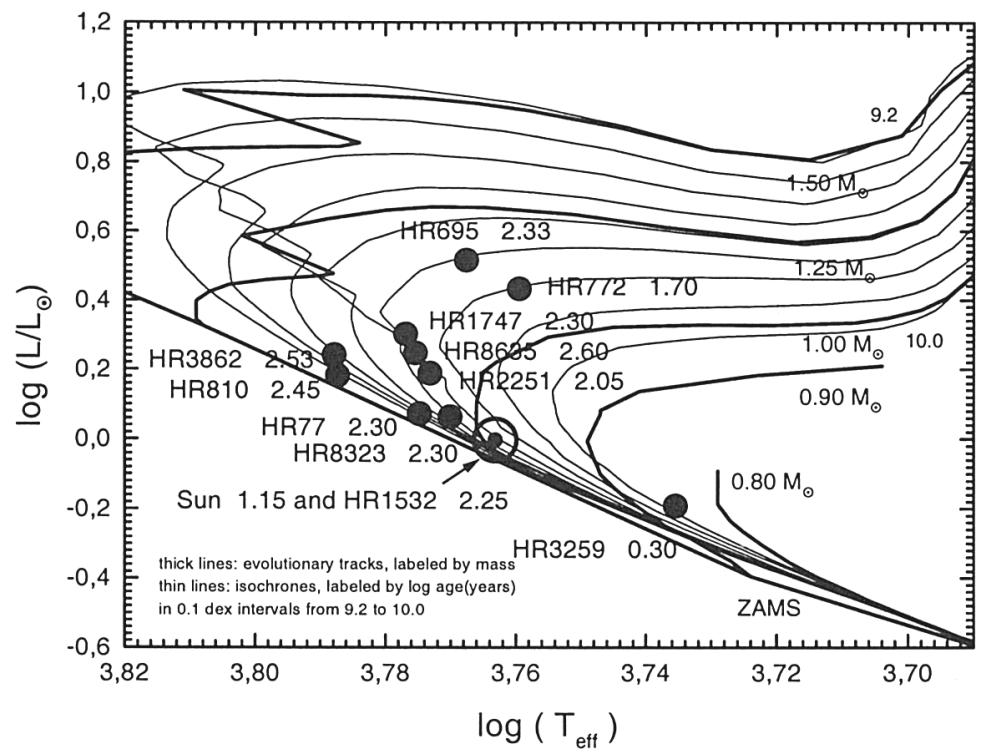

star dredges up to the surface Li that has been preserved below the convectively unstable surface layers, or else by the fact that these stars have maintained their $\mathrm{Li}$ abundance owing to low levels of depletion while on the main sequence (Randich et al. 1999). We have found that the metal-poor stars that are not yet subgiants are appreciably more Li-poor than their metal-richer peers. This may be explained by their being quite old stars: all of them lie in a narrow mass interval $\left(0.80-0.90 \mathrm{M}_{\odot}\right)$. They seem to have undergone different histories of $\mathrm{Li}$ depletion even among themselves, judging by their large $\mathrm{Li}$ abundance dispersion. The subgiant metal- rich and metal-normal stars have high to moderately high $\mathrm{Li}$ content, but the two metal-poor subgiants have much lower $\mathrm{Li}$ abundances. Whether they represent stars for which no "ressurgence" phenomenon was at work, or simply stars which depleted their Li while still close to the main-sequence, may not be decided with our current understanding.

\section{References}

Dravins D., Lindegren L., Nordlund A., Vandenberg D. A. 1993, ApJ, 403, 385 Habets G. M. J., Heintze J. R. W. 1981, A\&AS, 46, 193

King J. R., Deliyannis C. P., Hiltgen D. D., Stephens A., Cunha K., Boersgaard A. M. 1997, AJ, 113, 1871

Pasquini L., Liu Q., Pallavicini R. 1994, A\&A, 287, 191

Randich R., Gratton R., Pallavicini R., Pasquni L., Carretta E. 1999, A\&A, 348,487

Schaerer D., Meynet G., Maeder A., Schaller G. 1993, A\&AS, 98, 253 